\title{
Resource Theories of Nonclassical Light
}

\author{
Kok Chuan Tan * (D) and Hyunseok Jeong * \\ Department of Physics and Astronomy, Seoul National University, Seoul 08826, Korea \\ * Correspondence: bbtankc@gmail.com (K.C.T.); h.jeong37@gmail.com (H.J.)
}

Received: 12 September 2019; Accepted: 24 September 2019; Published: 26 September 2019

Abstract: In this focused review we survey recent progress in the development of resource theories of nonclassical light. We introduce the resource theoretical approach, in particular how it pertains to bosonic/light fields, and discuss several different formulations of resource theories of nonclassical light.

Keywords: quantum optics; nonclassicality; quantum resource theories; non-Gaussianity

\section{Introduction}

In quantum optics, there is general agreement that the most classical states of light are the coherent states [1-4]. In classical physics, a system can be completely described by a single point in phase space. In quantum mechanics, this is not possible due to the uncertainty principle $[5,6]$. For this reason, the phase space description of quantum systems must necessarily be via some distribution, rather than a point, that strictly obeys the uncertainty principle. In this sense the coherent state, being a minimum uncertainty state with no preferred axial direction in phase space, is arguably the closest quantum mechanical equivalent to a classical single point description of a physical system. However, one has to bear in mind that coherent states still fundamentally represent a quantum mechanical system. For this reason, despite being the most classical among the quantum states, quantum mechanical properties may still be pried from it. This is demonstrated by their strict observance of the uncertainty principle, as well as by their continued usefulness in purely quantum protocols such as quantum key distribution [7].

Nonclassical states, which are quantum states whose properties cannot be purely interpreted as directly deriving from the set of coherent states, are of considerable theoretical and practical interest. Fundamentally, because the set of classical states occupies only a small corner of the full Hilbert space, we are motivated to fully explore the set of physically allowable quantum states and to study their properties. Practically, there are many applications where nonclassical states can demonstrate genuine superiority over classical ones. These include applications in quantum information [8], quantum metrology [4], biology [9] and imaging [10]. It is also frequently the case that strongly nonclassical states are more useful in many protocols than weakly nonclassical ones. We are therefore interested in formulating methods that will enable us to first of all identify correctly when a light field can be considered nonclassical, as well as quantitatively describe the extent of nonclassicality present in quantum systems.

A recent trend in quantum information circles is to study different notions of nonclassicality via the so-called resource theoretical framework [11]. Such frameworks remove some of the ambiguity and arbitrariness in earlier studies of nonclassicality, by interpreting nonclassicality as resources to overcome the limitations of some set of quantum operation. Any quantifier of nonclassicality within this framework therefore has to be consistent with this view. This article seeks to provide some background to this approach, and to summarize the recent developments in this arena. 


\section{Preliminaries}

As mentioned, the most classical states of light are typically considered to be the coherent states. One way to define the coherent states is as the eigenvectors $|\alpha\rangle$ of the annihiliation operator $a$ such that

$$
a|\alpha\rangle=\alpha|\alpha\rangle
$$

where $\alpha$ is any complex number. If one accepts that among pure states $|\alpha\rangle$ is classical, then classical statistical mixtures of such states should also be classical. This suggests that if the density operator of a general mixed can be expressed in the form

$$
\rho_{\mathrm{cl}}=\int \mathrm{d}^{2} \alpha P_{\mathrm{cl}}(\alpha)|\alpha\rangle\langle\alpha|,
$$

where $\int \mathrm{d}^{2} \alpha P_{\mathrm{cl}}(\alpha)=1$ is some positive probability density function, then we can say that the quantum state $\rho$ is classical. We see that for classical states, the quantum state is fully described by the distribution $P_{\mathrm{cl}}$.

It turns out that for general mixed states, a similar representation of the state is also possible. Due to the seminal work of Glauber [2] and Sudarshan [3], one may show that every quantum state of light may be written in the form

$$
\rho=\int \mathrm{d}^{2} \alpha P(\alpha)|\alpha\rangle\langle\alpha|,
$$

where $P(\alpha)$ is called the Glauber-Sudarshan $P$-function. Observe the close similarity to the definition of a classical state $\rho_{\mathrm{cl}}=\int \mathrm{d}^{2} \alpha P_{\mathrm{cl}}(\alpha)|\alpha\rangle\langle\alpha|$. For general quantum states, however, the function $P(\alpha)$ is a quasiprobability distribution instead of a proper, positive probability density function. This means that $P(\alpha)$ is always properly normalized such that $\int \mathrm{d}^{2} \alpha P(\alpha)=1$, but may permit negative values. We see that when $P(\alpha)$ corresponds to a positive probability density function the state must be classical. Otherwise, we say that the state is nonclassical.

\section{The Resource Theoretical Framework}

A quantum resource theory [11] is a general framework for quantifying notions of nonclassicality. We stress that this approach is not limited to the study of nonclassicality in light fields. There are many different kinds of quantum resource theories that are currently being considered, such as the resource theories of entanglement [12] and coherence [13]. One benefit of studying different notions of nonclassicality under a unifying framework is that it also provides a method of considering the relationships between different quantum resources, rather than treating each different quantum resource as its own separate island.

While the underlying notion of nonclassicality may differ from resource theory to resource theory, the fundamental approach remains broadly the same. The idea is to cast different notions of quantumness as resources that are not freely available. If one has access of the full repertoire of quantum mechanical operations, such limitations are not possible. For this reason, a fundamental requirement of a resource theory is to first define a set of "free" quantum operations.

We now describe this in more precise terms. Let the set of classical states be $\mathcal{C}$, which is a strict subset of the Hilbert space. Any state that belongs to the complement of the set $\mathcal{C}$, i.e., does not belong to $\mathcal{C}$, is nonclassical by definition. Associated with the set of classical states $\mathcal{C}$, we will also define a set of operations $\mathcal{O} . \mathcal{O}$ must be a strict subset of the set of all possible quantum operations, with the only requirement being that if $\Phi \in \mathcal{O}$ and $\rho \in \mathcal{C}$, then $\Phi(\rho) \in \mathcal{C}$. In other words, $\mathcal{O}$ must be some collection of quantum operations that are unable to produce nonclassical states from classical ones.

Given the set of classical states $\mathcal{C}$ and the set of free operations $\mathcal{O}$, we are then in the position to formulate our resource theory. We shall say that a nonnegative measure $N(\rho)$ is a nonclassicality measure under a particular resource theory if it satisfies the following basic properties: 
1. $N(\rho)=0$ if $\rho \in \mathcal{C}$.

2. (Monotonicity) $N(\rho) \geq N(\Phi(\rho))$ if $\Phi \in \mathcal{O}$.

3. (Convexity), i.e., $N\left(\sum_{i} p_{i} \rho_{i}\right) \leq \sum_{i} p_{i} N\left(\rho_{i}\right)$.

Property 1 simply requires that the measure $N(\rho)$ returns positive values only when $\rho$ is nonclassical. Property 3 imposes the condition that $N(\rho)$ must be a convex function of state. This is a natural requirement to ensure that you cannot increase nonclassicality by statistically mixing any two quantum states $\rho$ and $\sigma$ and considering $p \rho+(1-p) \sigma$. It is intuitively clear that such statistical mixing processes are inherently classical procedures, and so cannot be expected to produce additional nonclassicality in any reasonable measure $N$.

Property 2 is perhaps the primary property that defines the resource theoretical approach. It imposes the requirement that any nonclassicality measure $N(\rho)$ must always be a nonclassicality monotone with respect to the set of operations $\mathcal{O}$. This means that the nonclassicality measure $N(\rho)$ must always monotonically decrease under the quantum operation $\Phi$, if $\Phi \in \mathcal{O}$. This property encapsulates the idea that one can neither freely produce nor increase nonclassicality if you are limited to performing only quantum operations that belong to the set $\mathcal{O}$. Suppose $N(\rho)$ is some physically relevant quantity that we are trying to maximize. There is clearly no way to do so by solely performing operations in $\mathcal{O}$ so the only way to increase $N(\rho)$ is by replacing the input state $\rho$ with another nonclassical state $\sigma$ such that $N(\sigma)>N(\rho)$. In this picture, sources of nonclassical quantum states may be interpreted as additional quantum resources that are required in order to overcome the limitations inherent to classical states $\mathcal{C}$ and operations $\mathcal{O}$.

We stress that the above is only a framework and does not constitute a physical result on its own. Physics comes into the picture by studying specific physical scenarios and then studying the properties of physically meaningful measures $N(\rho)$ under various conditions, so it is imperative that $N(\rho)$ has physically meaningful interpretations in the first place. What this framework does is only to imbue the quantity $N(\rho)$ with an additional resource interpretation, in the sense that we have described in the previous paragraph.

For the quantification of nonclassicality in light, the set of classical states is unambiguous: $\mathcal{C}$ must be the set of states with classical $P$-functions. The set of operations $\mathcal{O}$ therefore needs to be defined in order to formulate a resource theory of nonclassicality. The earliest known proposal to formulate a resource theory of nonclassicality for light is by Gehrke et al. [14,15]. In this resource theory, the set of free quantum operations is abstract. Subsequently, resource theories based on more physically relevant sets of free operations were also considered. We will discuss these various approaches in the subsequent sections.

\section{Resource Theory Based on Abstract Free Operations}

An early proposal to formulate a resource theory of nonclassicality for light is by Gehrke et al. [14,15]. In this proposal the set of free operations $\mathcal{O}$ is proposed to be the maximal set of quantum operations $\Phi$ that always maps a classical state to another classical state, i.e., $\Phi(\rho) \in \mathcal{C}$ if $\Phi \in \mathcal{O}$ for every $\rho \in \mathcal{C}$. This is the largest possible set of quantum operations that one can define under the resource theoretical approach.

One example of a nonclassicality measure under this resource theory is the nonclassicality degree. The nonclassicality degree is a discrete measure of nonclassicality, similar to the Schmidt number $[16,17]$ in the resource theory of entanglement. The set of coherent states is well known to form an overcomplete basis. This means that any pure state can be written in terms of a superposition of coherent states. As a result, we can consider the minimum number of superpositions $r$ such that

$$
|\psi\rangle=\sum_{i=1}^{r} \lambda_{i}\left|\alpha_{i}\right\rangle,
$$


where $\left\{\left|\alpha_{i}\right\rangle\right\}$ is some set of coherent states. The nonclassicality degree [14] is then defined as

$$
\kappa(|\psi\rangle):=r-1 .
$$

Since the only classical pure states are the coherent states, for any nonclassical pure state we must have $\kappa(|\psi\rangle) \geq 0$.

For mixed states, we can extend the measure for pure states via a convex roof construction $[18,19]$. This means that we consider all possible pure state decompositions $\left\{p_{i},\left|\psi_{i}\right\rangle\right\}$ such that $\rho=\sum_{i} p_{i}\left|\psi_{i}\right\rangle\left\langle\psi_{i}\right|$. The nonclassicality degree of some density operator $\rho$ can then be defined as the minimax quantity

$$
\kappa(\rho):=\min _{\left.\left\{p_{i}, \psi_{i}\right\rangle\right\}} \max _{i} \kappa\left(\left|\psi_{i}\right\rangle\right) .
$$

This is basically the largest nonclassicality degree $\kappa\left(\left|\psi_{i}\right\rangle\right)$, minimized over all possible pure state decompositions $\left\{p_{i},\left|\psi_{i}\right\rangle\right\}$.

Another example of a nonclassicality measure under this resource theory is the nonclassicality distance. The nonclassicality distance is actually a family of geometric nonclassicality measures rather than a single measure. Suppose we have a distance measure $d\left(\rho_{1}, \rho_{2}\right)$ for any quantum states $\rho_{1}$ and $\rho_{2}$. We can define the nonclassicality distance as

$$
\delta(\rho):=\inf _{\sigma_{\mathrm{cl}}} d\left(\rho, \sigma_{\mathrm{cl}}\right)
$$

In particular, some distance measures are known to be contractive under arbitrary completely positive, trace preserving (CPTP) quantum maps $\Phi$. This means that for every $\rho_{1}, \rho_{2}$ and CPTP map $\Phi$, we have $d\left(\rho_{1}, \rho_{2}\right) \geq d\left(\Phi\left(\rho_{1}\right), \Phi\left(\rho_{2}\right)\right)$. If the distance measure $d$ has such a property then we see that if $\Phi \in \mathcal{O}$ is a free operation, then

$$
\begin{aligned}
\delta(\rho) & :=\inf _{\sigma_{\mathrm{cl}}} d\left(\rho, \sigma_{\mathrm{cl}}\right) \\
& \geq \inf _{\sigma_{\mathrm{cl}}} d\left(\Phi(\rho), \Phi\left(\sigma_{\mathrm{cl}}\right)\right) \\
& \geq \delta(\Phi(\rho)) .
\end{aligned}
$$

We are therefore guaranteed that $\delta(\rho)$ monotonically decreases under free operations. If the distance measure $d$ is additionally convex with respect to its arguments, then Properties 1, 2, and 3 will always be satisfied. Examples of distance measures satisfying the required properties are the trace distance [20] and the Bures distance [21]. The trace distance is defined as

$$
d_{\operatorname{Tr}}\left(\rho_{1}, \rho_{2}\right):=\left\|\rho_{1}-\rho_{2}\right\|_{1},
$$

where $\|A\|_{1}:=\operatorname{Tr}\left(\sqrt{A^{+} A}\right)$ is the trace norm. The Bures distance is defined as

$$
d_{\mathrm{BU}}(\rho, \sigma):=\sqrt{2-2 F(\rho, \sigma)}
$$

where $F(\rho, \sigma):=\operatorname{Tr}\{\sqrt{\sqrt{\rho} \sigma \sqrt{\rho}}\}$ is the Bures fidelity. One notable example of a distance measure that does not possess the required properties is the Hilbert-Schmidt distance, which is known to be not contractive under general CPTP maps [22].

The primary issue with Gehrke et al.'s resource theory is how to interpret this abstract definition of the set of free operations $\mathcal{O}$. While simple to define, there is no known simple characterization of $O$ and it is unclear what kind of operations they represent physically in the laboratory. For the resource theoretical approach, this may not be always desirable, since one of the primary motivations behind this approach is to consider nonclassicality in terms of resource states that overcomes the 
limitations of the free operations $\mathcal{O}$. From this perspective, there is no strong physical motivation in Gehrke et al.'s approach as to why one should be interested to utilize nonclassical resources to overcome such abstract definitions of $\mathcal{O}$, beyond the fact that it is technically allowable under the resource theoretical framework.

\section{Resource Theory Based on Linear Optical Operations}

Recently, Tan et al. [23], noting that nonlinear operations are required in order to produce nonclassical states, proposed a resource theory of nonclassicality based on the set of linear optical operations. This approach is valid since linear optical operations always map a classical state to another classical state. This resolves the main problem with Gehrke et al.'s resource theory by replacing an abstract definition of $\mathcal{O}$ with a physically relevant set of operations that can be performed in the laboratory, and which also permits a relatively simple characterization.

In simple terms, a linear optical operation is any quantum operation that is implementable using any combination of passive linear optical elements, displacement operations, plus interaction with classical ancillas. The most fundamental operation in a general linear optical operation is a unitary linear optical operation $U_{L}$ representing any combination of beam splitters, mirrors, and phase shifters, supplemented by displacement operations. A general linear optical unitary operation $U_{L}$ can be characterized rather simply. Let $a_{k}^{+}$be the creation operator of the $k$ th mode, then $U_{L}$ represents any transformation of the type

$$
a_{i}^{\dagger} \rightarrow \sum_{k=1}^{K} \mu_{k} a_{k}^{\dagger}+\bigoplus_{k=1}^{K} \alpha_{k} I_{k}
$$

where $\left\{\mu_{k}\right\}$ are any set of complex values satisfying $\sum_{k=1}^{K}\left|\mu_{k}\right|^{2}=1, I_{k}$ are identity operators on the $k$ th mode, and $\alpha_{k}$ are complex numbers. More generally, a linear optical map is defined to be any map $\Phi_{L}$ that can be represented by the expression

$$
\Phi_{L}\left(\rho_{A}\right)=\operatorname{Tr}_{E}\left(U_{L} \rho_{A} \otimes \sigma_{E} U_{L}^{\dagger}\right),
$$

where $\sigma_{E}$ is some classical state. By defining $\mathcal{O}$ to be the set of linear optical maps, we can see that $\mathcal{O}$ is not only simple to define, it also has a clear physical interpretation.

One example of a measure under this resource theory is the amount of coherent superposition between the coherent states [23]. By decomposing a state $|\psi\rangle$ as a superposition of a carefully chosen set of coherent states $\left|\alpha_{i}\right\rangle$ such that $|\psi\rangle=\sum_{i} c_{i}\left|\alpha_{i}\right\rangle$, one can take a continuous coherence measure $C$ from the resource theory of coherence and transform it to a nonclassicality measure $N_{C}(|\psi\rangle)$ which quantifies the amount of coherent superposition among the set of coherent states $\left|\alpha_{i}\right\rangle$ according to the coefficients $c_{i}$. In Ref. [23] $N_{C}(|\psi\rangle)$ is referred to as the $\alpha$-coherence. One may show that the $\alpha$-coherence satisfies the required Properties 1,2 and 3 under the resource theory of Tan et al. so it is a valid nonclassicality quantifier. The measure $N_{C}$ may be interpreted as a continuous extension of the nonclassical degree Ref. [14], which is a discrete measure that counts the minimum number of superpositions rather than the amount of superposition. The $\alpha$-coherence therefore provides a neat interpretation of optical nonclassicality in terms of the resource theory of coherence [13]. In fact, it was also noted [23] that the nonclassicality of optical systems shares many points of similarities with quantum coherence. For instance, both nonclassicality [24-26] and coherence [27-30] may be converted into entanglement using only free operations. The relationship between entanglement, coherence and nonclassicality may be an interesting point of investigation going forward.

Another measure that belongs to the resource theory by Tan et al. is called the metrological power. It is based on the quantum Fisher information, which quantifies the usefulness of a quantum probe for parameter estimation tasks. This measure was considered independently in both Ref. [31] and Ref. [32] with the former focusing on pure states, and the latter with a greater focus on general mixed states.

We now introduce the quantum Fisher information and discuss its relationship to parameter estimation. Suppose we have some unitary $U_{\theta}:=e^{-i \theta G}$, where $\theta$ is a real number representing 
some physical parameter, and $G$ is some Hermitian operator called the generator. Under this unitary evolution, an initial state $\rho$ will evolve according to $\rho \rightarrow \rho_{\theta}=U_{\theta} \rho U_{\theta}^{\dagger}$. The primary goal of parameter estimation is to estimate the value of $\theta$ by performing a measurement on $\rho_{\theta}$.

The relationship between parameter estimation and the Fisher information is supplied by the well known quantum Cramér-Rao bound [33-35]. This states that the uncertainty of any unbiased estimator $\hat{\theta}$ must obey the inequality

$$
\Delta^{2} \hat{\theta} \geq \frac{1}{n I_{Q}(\rho, G)},
$$

where $n$ refers to the number of independent measurements, and $I_{Q}(\rho, G)$ is the quantum Fisher information given by $I_{Q}(\rho, G)=2 \sum_{i, j} \frac{\left(p_{i}-p_{j}\right)^{2}}{p_{i}+p_{j}}|\langle i|G| j\rangle|^{2}$. It is known that for single parameter estimation problems, there always exists a quantum measurement that saturates this bound, so long as a sufficient number of independent measurements $n \gg 1$ is performed. The quantum Fisher information $I_{Q}(\rho, G)$ therefore sets fundamental limits on our ability to measure $\theta$.

Consider now a system of $N$ modes with creation and annihilation operators $a_{i}^{\dagger}$ and $a_{i}$ where $i=1, \ldots, N$. A $N$ mode annihilation operator can be defined as $a_{\mu}:=\sum_{i=1}^{N} \mu_{i} a_{i}$ where $\boldsymbol{\mu}=\left[\operatorname{Re}\left(\mu_{1}\right), \operatorname{Im}\left(\mu_{1}\right) \ldots, \operatorname{Re}\left(\mu_{N}\right), \operatorname{Im}\left(\mu_{N}\right)\right]$ is a $2 N$ dimensional real vector of unit length, i.e., $|\boldsymbol{\mu}|^{2}=\sum_{i=1}^{N}\left|\mu_{i}\right|^{2}=1$. From this, we define the $N$ mode field quadrature operator

$$
X_{\mu}:=\frac{a_{\mu}+a_{\mu}^{\dagger}}{\sqrt{2}} .
$$

$X_{\mu}$ is the generator of the the unitary evolution

$$
D(\theta, \mu):=e^{-i \theta X_{\mu}},
$$

which performs an $N$ mode displacement operation. Setting $G=X_{\mu}$, we can consider the Fisher information:

$$
I_{Q}\left(\rho, X_{\mu}\right)=2 \sum_{i, j} \frac{\left(p_{i}-p_{j}\right)^{2}}{p_{i}+p_{j}}\left|\left\langle i\left|X_{\mu}\right| j\right\rangle\right|^{2} .
$$

Generally speaking, the larger the Fisher information, the more useful it is for estimating the parameter $\theta$ in $D(\theta, \boldsymbol{\mu})$, so we are interested to find the maximum Fisher information that we can extract from a quantum probe $\rho$ over all possible quadrature directions given by $\mu$, so we can consider for our figure of merit

$$
M(\rho):=\max \left\{\frac{1}{2} \max _{\mu \in S} I_{Q}\left(\rho, X_{\mu}\right)-1,0\right\},
$$

where the second maximization ensures the non-negativity of the measure. In the first maximization, $S$ is simply the set of all possible $\mu$ satisfying $|\boldsymbol{\mu}|^{2}=1 . M(\rho)$ is called the metrological power and can be shown to satisfy Properties 1,2, and 3 under the resource theory of Tan et al. The interesting aspect of the metrological power is that it directly relates the nonclassicality of a state to the state's usefulness in a parameter estimation task. For pure states, the metrological power can identify every nonclassical state, thereby establishing that every nonclassical pure state is useful in this particular parameter estimation problem, and the usefulness of the state is directly related to the amount of nonclassicality. One limitation is that the metrological power does not identify every mixed quantum state, i.e., there may be some mixed, nonclassical states where the measure returns zero.

More recently, Ref. [36] considered the extension of negativity to cover the set of all s-parametrized quasiprobabilities $P_{s}(\alpha)$. The $s$-parametrized quasiprobabilities where $s \in[-1,1]$ are a family of phase space distribution functions satisfying $\int \mathrm{d}^{2} \alpha P_{s}(\alpha)=1$ but may possess negativities. Setting $s=1$ 
we get the Glauber-Sudarshan $P$-function, setting $s=0$ we retrieve the Wigner function, and setting $s=-1$ we get the Husimi $Q$-function. One may show that the negativity of all such distributions

$$
N_{\mathcal{S}}(\rho):=\frac{1}{2}\left[\int \mathrm{d}^{2} \alpha\left|P_{\mathcal{S}}(\alpha)\right|-1\right]
$$

are nonclassicality measures belonging to the resource theory of Tan et al. [23]. In particular, as $s$ decreases, $N_{s}(\rho)$ becomes increasingly weaker as a nonclassicality measure in the sense that the negativity decreases and fewer nonclassical states are identified by the measure.

In Ref. [31], Yadin et al. also considered a resource theory where $\mathcal{O}$ is expanded to include the set of linear optical operations, plus operations allowing for the feed forward of measurement outcomes. We note that, by definition, linear optical operations belong to this expanded set of operations. As such, any measure of nonclassicality under the resource theory of Yadin et al. [31] will monotonically decrease under linear optical operations and thus also belongs to the resource theory of Tan et al. [23]. Similar arguments can also be made for the resource theory of Gehrke et al. [14,15].

\section{Convex Resource Theories of Non-Gaussianity}

A Gaussian state is a special class of optical quantum states whose Wigner function is a Gaussian function [37]. According to this definition, every Gaussian state $\rho_{\mathrm{G}}$ has a Wigner function that can be written in the form

$$
W(\mathbf{r})=\frac{1}{2 \pi \sqrt{\operatorname{det} V}} \exp \left[-\frac{1}{2}(\mathbf{r}-\overline{\mathbf{r}})^{T} V^{-1}(\mathbf{r}-\overline{\mathbf{r}})\right],
$$

where $\overline{\mathbf{r}}:=(\langle x\rangle,\langle p\rangle)$ and $V$ is the covariance matrix, which is given by

$$
\left[\begin{array}{cc}
\Delta^{2} x & \left\langle\frac{1}{2}\{x-\langle x\rangle, p-\langle p\rangle\}\right\rangle \\
\frac{1}{2}\langle\{x-\langle x\rangle, p-\langle p\rangle\}\rangle & \Delta^{2} p
\end{array}\right] .
$$

Quantum states such as coherent states, thermal states, and squeezed vacuum states are Gaussian. We observe that the coherent and thermal states are both classical states, while squeezed vacuum is an example of a nonclassical Gaussian state.

Based on the above definition of Gaussian states, we can also define the set of Gaussian operations. A Gaussian unitary $U_{\mathrm{G}}$ is any combination of displacement operations, phase shifters, beam splitters and squeezing operations [38,39]. More generally, Gaussian operations $\Phi_{\mathrm{G}}$ can be written in the form

$$
\Phi_{\mathrm{G}}\left(\rho_{1}\right)=\operatorname{Tr}_{2}\left[U_{\mathrm{G}} \rho_{1} \otimes|0\rangle\left\langle\left. 0\right|_{2} U_{\mathrm{G}}^{\dagger}\right] .\right.
$$

We see that such maps always map a Gaussian state to another Gaussian state. Many Gaussian states can also be produced under laboratory settings [8]. As a result, the properties of Gaussian states are particularly well understood. At the same time, Gaussian states comprise only a small subset of the possible quantum states, and many quantum protocols are not possible if one stays strictly within the Gaussian regime [40-49]. This has motivated the study of non-Gaussian states as quantum resources, so there is considerable interest to quantify and characterize non-Gaussian effects.

In the strictest sense, every quantum state whose Wigner function is not a Gaussian distribution is non-Gaussian by definition. One may try to formulate non-Gaussianity measures based on this definition. Examples of such measures are the geometric based measures similar to the nonclassicality distance [50-55] which quantifies the distance of a state $\rho$ to the closest Gaussian state $\rho_{\mathrm{G}}$. However, employing this strict definition necessarily means that many non-Gaussian states are classical. An example of this is the mixed coherent state $\rho=\left(\left|\alpha_{1}\right\rangle\left\langle\alpha_{1}|+| \alpha_{2}\right\rangle\left\langle\alpha_{2}\right|\right) / 2$, which is clearly classical but has two peaks and cannot be written in the form of Equation (4). Such states can be created using only classical states and classical statistical processes, and should not lead to any genuine quantum effects. 
In order to circumvent this, there are recent proposals to formulate a convex quantum resource theory of non-Gaussianity [56,57] where genuine nonclassical effects are captured. In such proposals, the definition of non-Gaussianity is now stricter, and only includes any quantum state that is not within the convex hull of Gaussian states. Such states are said to display genuine non-Gaussianity, which means that non-Gaussianity that emerges from classical statistical mixing processes is now excluded. Such genuinely non-Gaussian states cannot be written in the form

$$
\rho=\sum_{i} p_{i} \rho_{G}^{i}
$$

where $p_{i}$ is a probability distribution and $\rho_{G}^{i}$ is some Gaussian state. Since coherent states are Gaussian, every state with a classical $P$-function will lie within the convex hull of Gaussian states. As such, genuinely non-Gaussian resource states must also have nonclassical $P$-functions.

In such resource theories, the set of classical states $\mathcal{C}$ is therefore the set of states that can be written in the form $\rho=\sum_{i} p_{i} \rho_{G}^{i}$. As for the set of free operations $\mathcal{O}$, one possibility is to define it as any quantum operation $\Phi$ that is composed of the following elementary operations:

a. Gaussian unitary operations $U_{G}$.

b. Composition with a free state $\rho \otimes \sigma$, where $\sigma=\sum_{i} p_{i} \sigma_{G}^{i} p_{i}$ is some probability distribution, and $\sigma_{G}^{i}$ is some Gaussian state.

c. Partial trace of a subsystem.

In addition to the above elementary operations, the resource theories of Ref. [56] and Ref. [57] also considered Gaussian operations conditioned on measurement outcomes. In this respect, their definition of a free operation differs slightly in that the former considered projective measurements onto general Gaussian pure states $\left|\psi_{G}\right\rangle\left\langle\psi_{G}\right|$ while the latter considered homodyne measurements.

One example of a genuine non-Gaussianity measure is the negativity of the Wigner function [56,57]. Given the Wigner function $W(\mathbf{r})$ of some given state $\rho$, the logarithmic negativity of $\rho$ is defined as

$$
L_{w}(\rho):=\log \int \mathrm{d}^{2} \mathbf{r}|W(\mathbf{r})| .
$$

Recall from Equation (3) that the family of negativity measures $N_{S}$ are all nonclassicality measures. Setting $s=0, N_{0}$ is nothing more than the negativity of the Wigner function, so we have $\log \left[2 N_{0}(\rho)+1\right]=L_{w}(\rho)$. We therefore see that the negativity of the Wigner function is both a measure of genuine non-Gaussianity and nonclassicality.

In fact, every convex measure of genuine non-Gaussianity must necessarily be a nonclassicality measure. This is because coherent states are Gaussian states, and every linear optical unitary operation is also a Gaussian preserving unitary operation. From Properties a, b and c, we see that any linear optical operation of the type in Equation (1) must also be a free operation in the convex resource theory of non-Gaussianity. This implies that measures of genuine non-Gaussianity must also be linear optical monotones in the resource theory of Tan et al. [23].

We note that in such convex resource theories of non-Gaussianity, the convex hull of Gaussian states $\mathcal{C}$ necessarily contains many states that have nonclassical $P$-functions, with the most prominent being the squeezed coherent states. Any genuine non-Gaussianity measure will therefore exclude such states. Furthermore, since the squeezing operation is a Gaussian operation, it makes no attempt to capture any increase in nonclassicality due to squeezing. This is a feature that stems from the definition of the resource theory. As such, the starting point of the resource theory of non-Gaussianity is necessarily qualitatively different from resource theory of nonclassicality discussed in the preceding sections. For this reason, it is perhaps more appropriate to consider the concept of genuine non-Gaussianity as something that overlaps significantly with the notion of nonclassicality, rather than an independent approach of quantifying nonclassicality in light. 


\section{Conclusions}

In this article we summarized recent trends in the characterization and quantification of nonclassical states of light. Along these lines, there has been considerable interest in developing a resource theory of nonclassicality for quantum light. Several prominent proposals are discussed. First, there is the resource theory of Gehrke et al. [14,15], which is based on an abstract definition of the set of free operations $\mathcal{O}$. We then discuss the resource theories of Tan et al. [23] and Yadin et al. [31], which are based on linear optical operations. Finally, we discuss proposals by Albarelli et al. [56], and Takagi and Zhuang [57] to develop convex resource theories of non-Gaussianity. Non-Gaussianity approaches are not technically resource theories of nonclassicality as the set of free states are not the set of states with classical $P$-functions, but there exists significant overlap.

It is interesting to note that all the measures discussed across these various schemes naturally fall under the resource theory of Tan et al. [23]. This is because the free operations under this resource theory, the set of linear optical operations, is the simplest among the resource theories that are discussed thus far.

Most of the nonclassicality measures that belong to these resource theories have geometric or other fundamental interpretations, with the exception of the metrological power [32], $M(\rho)$, which has an interpretation in terms of the operational usefulness of the state. This measure is sufficient to identify every nonclassical pure state as useful for a parameter estimation task, but does not identify every nonclassical mixed state in general. It is an open problem whether there is some parameter estimation problem that can identify every nonclassical state as useful. More generally, a promising future direction is to find nonclassicality measures that directly quantify the state's usefulness in some meaningful operational task.

Author Contributions: Writing—original draft, K.C.T.; Writing—review \& editing, K.C.T. and H.J.

Funding: This work was supported by the National Research Foundation of Korea (NRF) through a grant funded by the the Ministry of Science and ICT (Grant No. NRF-2019R1H1A3079890). K.C. Tan was supported by Korea Research Fellowship Program through the National Research Foundation of Korea (NRF) funded by the Ministry of Science and ICT (Grant No. 2016H1D3A1938100).

Conflicts of Interest: The authors declare no conflict of interest.

\section{References}

1. Schrodinger, E. Der stetige Übergang von der Mikro- zur Makromechanik. Naturwissenschaften 1926, 14, 664-666. [CrossRef]

2. Glauber, R.J. Coherent and Incoherent States of the Radiation Field. Phys. Rev. 1963, 131, 2766. [CrossRef]

3. Sudarshan, E.C.G. Equivalence of Semiclassical and Quantum Mechanical Descriptions of Statistical Light Beams. Phys. Rev. Lett. 1963, 10, 277. [CrossRef]

4. Tan, K.C.; Jeong, H. Nonclassical Light and Metrological Power: An Introductory Review. arXiv. Available online: https: / / arxiv.org/abs/1909.00942 (accessed on 26 September 2019).

5. Heisenberg, Z.W. Über den anschaulichen Inhalt der quantentheoretischen Kinematik und Mechanik. Physic 1927, 43, 172.

6. Robertson, H.P. The Uncertainty Principle. Phys. Rev. 1929, 34, 163. [CrossRef]

7. Grosshans, F.; Assche, G.V.; Wenger, J.; Brouri, R.; Cerf, N.J.; Grangier, P. Quantum key distribution using gaussian-modulated coherent states. Nature 2003, 421, 238. [CrossRef] [PubMed]

8. Braunstein, S.L.; van Loock, P. Quantum information with continuous variables. Rev. Mod. Phys. 2005, 77, 513. [CrossRef]

9. Taylor, M.A.; Bowen, W.P. Quantum metrology and its application in biology. Phys. Rep. 2005, 615, 1. [CrossRef]

10. Berchera, I.R.; Degiovanni, I.P. Quantum imaging with sub-Poissonian light: challenges and perspectives in optical metrology. Metrologia 2019, 56, 024001. [CrossRef]

11. Chitambar, E.; Gour, G. Quantum resource theories. Rev. Mod. Phys. 2019, 91, 025001. [CrossRef] 
12. Horodecki, R.; Horodecki, P.; Horodecki, M.; Horodecki, K. Quantum entanglement. Rev. Mod. Phys. 2009, 81, 865. [CrossRef]

13. Streltsov, A.; Adesso, G.; Plenio, M.B. Colloquium: Quantum coherence as a resource. Rev. Mod. Phys. 2017, 89, 041003. [CrossRef]

14. Gehrke, C.; Sperling, J.; Vogel, W. Quantification of nonclassicality. Phys. Rev. A 2012, 86, 052118. [CrossRef]

15. Sperling, J.; Vogel, W. Convex ordering and quantification of quantumness. Phys. Scr. 2015, 90, 074024. [CrossRef]

16. Schmidt, E. Zur Theorie der linearen und nichtlinearen Integralgleichungen. Math. Ann. 1906, 63, 433. [CrossRef]

17. Terhal, B.M.; Horodecki, P. Schmidt number for density matrices. Phys. Rev. A 1999, 61, 040301. [CrossRef]

18. Bennett, C.H.; Di Vincenzo, D.P.; Smolin, J.A.; Wootters, W.K. Mixed-state entanglement and quantum error correction. Phys. Rev. A 1996, 54, 3824. [CrossRef]

19. Uhlmann, A. Optimizing entropy relative to a channel or a subalgebra. Open Syst. Inf. Dyn. 1998, 5, 209. [CrossRef]

20. Hillery, M. Nonclassical distance in quantum optics. Phys. Rev. A 1987, 35, 725. [CrossRef]

21. Marian, P.; Marian, T.A.; Scutaru, H. Quantifying Nonclassicality of One-Mode Gaussian States of the Radiation Field. Phys. Rev. Lett. 2002, 88, 153601. [CrossRef]

22. Ozawa, M. Entanglement measures and the Hilbert-Schmidt distance. Phys. Lett. A 2000, 268, 158. [CrossRef]

23. Tan, K.C.; Volkoff, T.; Kwon, H.; Jeong, H. Quantifying the Coherence between Coherent States. Phys. Rev. Lett. 2017, 119, 190405. [CrossRef] [PubMed]

24. Kim, M.S.; Son, W.; Bužek, V.; Knight, P.L. Entanglement by a beam splitter: Nonclassicality as a prerequisite for entanglement. Phys. Rev. A 2002, 65, 032323. [CrossRef]

25. Wang, X.-B. Theorem for the beam-splitter entangler. Phys. Rev. A 2002, 66, 024303.

26. Asbóth, J.K.; Calsamiglia, J.; Ritsch, H. Computable Measure of Nonclassicality for Light. Phys. Rev. Lett. 2005, 94, 173602. [CrossRef]

27. Streltsov, A.; Singh, U.; Dhar, H.S.; Bera, M.N.; Adesso, G. Measuring Quantum Coherence with Entanglement. Phys. Rev. Lett. 2015, 115, 020403. [CrossRef] [PubMed]

28. Tan, K.C.; Choi, S.; Kwon, H.; Jeong, H. Emergence of synchronization and regularity in firing patterns in time-varying neural hypernetworks. Phys. Rev. A 2018, 97, 052304. [CrossRef]

29. Tan, K.C.; Kwon, H.; Park, C.-Y.; Jeong, H. Unified view of quantum correlations and quantum coherence. Phys. Rev. A 2016, 94, 022329. [CrossRef]

30. Tan, K.C.; Jeong, H. Entanglement as the Symmetric Portion of Correlated Coherence. Phys. Rev. A 2018, 121, 220401. [CrossRef]

31. Yadin, B.; Binder, F.C.; Thompson, J.; Narasimhachar, V.; Gu, M.; Kim, M.S. Operational Resource Theory of Continuous-Variable Nonclassicality. Phys. Rev. X 2018, 8, 041038. [CrossRef]

32. Kwon, H.; Tan, K.C.; Volkoff, T.; Jeong, H. Nonclassicality as a Quantifiable Resource for Quantum Metrology. Phys. Rev. Lett. 2019, 122, 040503. [CrossRef] [PubMed]

33. Helstrom, C.W. Quantum Detection and Estimation Theory; Academic Press: Cambridge, MA, USA, 1976.

34. Holevo, A.S. Probabilistic and Statistical Aspects of Quantum Theory; Springer: Berlin, Germany, 1982.

35. Braunstein, S.L.; Caves, C.M. Statistical distance and the geometry of quantum states. Phys. Rev. Lett. 1994, 72, 3439. [CrossRef] [PubMed]

36. Tan, K.C.; Choi, S.; Jeong, H. Negativity of quasiprobability distributions as a measure of nonclassicality. arXiv. Available online: https:/ / arxiv.org/abs/1906.05579 (accessed on 26 September 2019).

37. Adesso, G.; Ragy, S.; Lee, A.R. Continuous variable quantum information: Gaussian states and beyond. Open Syst. Inf. Dyn. 2014, 21, 1440001. [CrossRef]

38. Ma, X.; Rhodes, W. Multimode squeeze operators and squeezed states. Phys. Rev. A 1990, 41, 4625. [CrossRef] [PubMed]

39. Cariolaro, G.; Pierobon, G. Bloch-Messiah reduction of Gaussian unitaries by Takagi factorization. Phys. Rev. A 2016, 94, 062109. [CrossRef]

40. Lloyd, S.; Braunstein, S.L. Quantum Computation over Continuous Variables. Phys. Rev. Lett. 1999, 82, 1784. [CrossRef]

41. Eisert, J.; Scheel, S.; Plenio, M.B. Distilling Gaussian States with Gaussian Operations is Impossible. Phys. Rev. Lett. 2002, 89, 137903. [CrossRef] 
42. Giedke, G.; Cirac, J.I. Characterization of Gaussian operations and distillation of Gaussian states. Phys. Rev. A 2002, 66, 032316. [CrossRef]

43. Fiurášek, J. Gaussian Transformations and Distillation of Entangled Gaussian States. Phys. Rev. Lett. 2002, 89, 137904. [CrossRef]

44. Bartlett, S.D.; Sanders, B.C. Universal continuous-variable quantum computation: Requirement of optical nonlinearity for photon counting. Phys. Rev. A 2002, 65, 042304. [CrossRef]

45. Cerf, N.J.; Krüger, O.; Navez, P.; Werner, R.F.; Wolf, M.M. Non-Gaussian Cloning of Quantum Coherent States is Optimal. Phys. Rev. Lett. 2005, 95, 070501. [CrossRef] [PubMed]

46. Menicucci, N.C.; van Loock, P.; Gu, M.; Weedbrook, C.; Ralph, T.C.; Nielsen, M.A. Universal Quantum Computation with Continuous-Variable Cluster States. Phys. Rev. Lett. 2006, 97, 110501. [CrossRef] [PubMed]

47. Niset, J.; Fiurášek, J.; Cerf, N.J. No-Go Theorem for Gaussian Quantum Error Correction. Phys. Rev. Lett. 2009, 102, 120501. [CrossRef] [PubMed]

48. Zhang, S.L.; van Loock, P. Distillation of mixed-state continuous-variable entanglement by photon subtraction. Phys. Rev. A 2010, 82, 062316. [CrossRef]

49. Ohliger, M.; Kieling, K.; Eisert, J. Limitations of quantum computing with Gaussian cluster states. Phys. Rev. A 2010, 82, 042336. [CrossRef]

50. Genoni, M.G.; Paris, M.G.A.; Banaszek, K. Measure of the non-Gaussian character of a quantum state. Phys. Rev. A 2007, 76, 042327. [CrossRef]

51. Genoni, M.G.; Paris, M.G.A.; Banaszek, K. Quantifying the non-Gaussian character of a quantum state by quantum relative entropy. Phys. Rev. A 2008, 78, 060303R. [CrossRef]

52. Genoni, M.G.; Paris, M.G.A. Quantifying non-Gaussianity for quantum information. Phys. Rev. A 2010, 82, 052341. [CrossRef]

53. Ivan, J.S.; Kumar, M.S.; Simon, R. A measure of non-Gaussianity for quantum states. Quantum Inf. Process. 2012, 11, 853. [CrossRef]

54. Ghiu, I.; Marian, P.; Marian, T.A. Measures of non-Gaussianity for one-mode field states. Phys. Scr. 2013, T153, 014028. [CrossRef]

55. Park, J.; Lee, J.; Ji, S.-W.; Nha, H. Quantifying non-Gaussianity of quantum-state correlation. Phys. Rev. A 2017, 96, 052324. [CrossRef]

56. Albarelli, F.; Genoni, M.G.; Matteo, M.G.A.; Ferraro, A. Resource theory of quantum non-Gaussianity and Wigner negativity. Phys. Rev. A 2018, 98, 052350. [CrossRef]

57. Takagi, R.; Zhuang, Q. Convex resource theory of non-Gaussianity. Phys. Rev. A 2018, 97, 062337. [CrossRef] 